\title{
Empoderamiento y demanda de autogestión. Estudio comparativo de emprendimientos de mujeres indígenas ${ }^{1}$
}

\author{
Empowerment and demand for self-management. \\ Comparative study of entrepreneurships of indigenous women
}

\author{
Gloria Mora Guerreroa, Daniela Meli Fernández ${ }^{b}$, Pilar Astete Ramos ${ }^{c}$ \\ a Académica de la Facultad de Ciencias de la Salud de la Universidad Católica de Temuco.

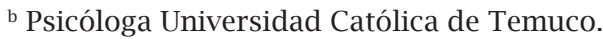 \\ ' Psicóloga Universidad Católica de Temuco. \\ gmora@uct.cl; dmeli2012@alu.uct.cl; eastete2012@alu.uct.cl
}

\section{RESUMEN}

En este artículo se describen las consecuencias de participar en un emprendimiento productivo desde la perspectiva de mujeres indígenas; $y$, en segundo lugar, se explora si en estas descripciones las participantes evidenciaron cuestionamientos a las inequidades de género, lo que podría haberse favorecido si las mujeres desarrollaron su emprendimiento en el contexto de un programa para su empoderamiento implementado con perspectiva de género. Para ello, se recurrió a un estudio cualitativo con enfoque intercultural y diseño comparativo que permitió comparar, por un lado, las experiencias de mujeres mapuche de Chile participantes de un emprendimiento surgido en el marco de un programa con perspectiva de género y, por otro, las experiencias de mujeres mayas de México, participantes de un emprendimiento bajo la dirección de un programa sin esta perspectiva. Los resultados mostraron que, más allá de si el emprendimiento tiene o no perspectiva de género, lo que parece tener implicaciones para el empoderamiento de las mujeres es si son ellas quienes autogestionan su emprendimiento.

PALABRAS CLAVE: Empoderamiento, Género, Mujeres indígenas, Emprendimiento, Autogestión.

\section{ABSTRACT}

In the first place, we described the consequences of participating in a productive entrepreneurship from the perspective of indigenous women; and, secondly, we explored

1 Proyecto de investigación financiado por la Dirección General de Investigación y Postgrado de la Universidad Católica de Temuco, Proyecto DGIPUCT 2015PF-GM-05, titulado "Género, políticas públicas de desarrollo rural y políticas culturales de reconocimiento. Una aproximación a la dinámica cultural en espacios de interfaz social en la IX Región". 
whether in these descriptions the women evidenced questions about gender inequities, which could have been favored if women developed their entrepreneurship in the context of a program for their empowerment implemented with a gender perspective. For this, a qualitative study with an intercultural approach and comparative design was used to compare, on the one hand, the experiences of Chilean Mapuche women participating in a project that emerged in the framework of a program with a gender perspective and, on the other, the experiences of Mayan women in Mexico, participants of an entrepreneurship under the direction of a program without this perspective. The results showed that, regardless of whether or not the entrepreneurship has a gender perspective, what seems to have implications for the empowerment of women is whether they are self-managing their entrepreneurship..

KEY WORDS: Empowerment, Gender, Indigenous women, entrepreneurship, selfmanagment

\section{INTRODUCCIÓN}

Recientes estudios han explorado las consecuencias de los programas de emprendimiento productivo sobre la autonomía personal, familiar y colectiva de las mujeres, incluyendo las mujeres indígenas (Fostner, 2013; Hoinle et al. 2013; Subramaniam, 2012). Tales programas operan sobre el supuesto de que existe una relación entre el emprendimiento económico de las mujeres y el fortalecimiento de su capacidad para ejercer el poder a nivel personal, colectivo y de relaciones cercanas, lo que en la teoría se ha definido como proceso de empoderamiento (Rowlands, 1997). No obstante, no se encontraron estudios que exploraran si la implementación de un programa de este tipo con perspectiva de género, es decir, con intención explícita de promover que las mujeres cuestionen las inequidades de género, tal como sugiere el enfoque de Género en el Desarrollo (GED) en contraposición al enfoque de Mujeres en el Desarrollo (MED) (Moser, 1991), promueve o no un mayor empoderamiento de las mujeres, sobre todo de aquellas que pertenecen a un pueblo indígena.

En este contexto, esta investigación se propuso describir, a la luz de las teorías del empoderamiento, las consecuencias personales, familiares y colectivas que tiene para las mujeres indígenas participar en emprendimientos productivos, y si marca alguna diferencia en términos de los cuestionamientos que las mujeres realizan a las inequidades de género el hecho de que su emprendimiento se implemente en el marco de un programa con o sin perspectiva de género. Las preguntas que se intentaron resolver fueron cuáles son las consecuencias a nivel personal, familiar y colectivo de participar en un emprendimiento productivo, desde el punto de vista de las mujeres participantes; $y$, si en las descripciones que realizan de dichas consecuencias, las mujeres evidencian cuestionamientos a las inequidades de género que pudieran relacionarse con la implementación de su emprendimiento bajo la dirección de un programa GED o MED. 
Para responder a estas preguntas, se seleccionaron dos casos de estudio. El primer caso comprendió a cinco participantes de un emprendimiento de mujeres mapuche de la comuna de Carahue, en la región de la Araucanía, en Chile, que opera en el marco de un programa que incorpora de forma explícita en sus lineamientos la perspectiva de género. En otras palabras, se trata de un programa GED, vale decir, un programa que promueve entre sus participantes el cuestionamiento a las relaciones de poder inequitativas entre mujeres y hombres (Moser, 1991). Por este motivo, para los fines de esta investigación, dicho programa se identificó como GED. El segundo caso lo constituyó un grupo de cinco mujeres emprendedoras mayas del municipio de Hunucma, en el estado de Yucatán, México, cuyo emprendimiento tiene lugar en el contexto de un programa que no asume la perspectiva de género como modo de operación, por lo que en esta investigación se entendió como programa MED.

Teóricamente, el trabajo implicó la referencia al concepto de empoderamiento. Los resultados del estudio podrían ser útiles al personal directivo y técnico de los programas de emprendimiento dirigidos a mujeres, pues potencian la reflexión respecto a la relación entre las características del programa y sus posibles consecuencias en términos del empoderamiento de las participantes.

\section{Emprendimiento, Empoderamiento y mujeres indígenas}

Desde los años noventa, los gobiernos, agencias de cooperación internacional y organizaciones no gubernamentales han impulsado la incorporación de los pueblos indígenas al desarrollo, lo que más tarde generó la identificación de la mujer indígena como un actor clave en la materia (Castelnuovo, 2013). Asimismo, el Fondo de Desarrollo de las Naciones Unidas para la Mujer (UNIFEM) se propuso como objetivo "empoderar" económicamente a los grupos de mujeres mediante alternativas viables y sostenibles para la erradicación de la pobreza, con lo cual se apoyaron iniciativas que impulsaron el desarrollo económico de grupos de mujeres por medio de la organización, la capacitación y la asignación de recursos materiales y financieros para la producción (Canorea, 2015).

Son dos los grandes enfoques desde los cuales se han implementado los emprendimientos dirigidos a mujeres. El primero es el enfoque de Mujeres en Desarrollo (MED), que impulsó emprendimientos en tres líneas: la equidad, que reconoce la posición de desigualdad en la que se encuentra la mujer; la antipobreza, que reconoce la desigualdad económica y propone más empleo y más ingresos para la mujer; y, por último, la eficacia, que promueve mayor participación económica en las mujeres (García, 2008). El segundo gran enfoque es el Género en el Desarrollo (GED), que advierte cómo las diferencias sociales entre hombres y mujeres se construyen a partir de las diferencias biológicas y se convierten en desigualdades sociales (Moser, 1991). Los programas que asumen este último enfoque buscan cambiar las condiciones materiales de hombres y mujeres y las relaciones de poder y control de los recursos materiales y no materiales (IICA, 1993), es decir, buscan que la mujer ejerza poder por sí misma o, en otras 
palabras, que se empodere. En términos generales, el debate entre ambos enfoques se reduce a la reflexión respecto a si las ganancias económicas son suficientes para empoderar a las mujeres, o bien, si se requiere que, a la vez que las mujeres aumentan sus ingresos, cuestionen las relaciones de género y las estructuras que las mantienen subordinadas (Young, 1997).

Respecto al empoderamiento, éste puede entenderse como el proceso por el cual aquellos quienes han permanecido excluidos de la posibilidad de tomar decisiones de vida estratégicas adquieren tal capacidad (Kabeer, 1999). Otros autores lo han definido como la capacidad de un sujeto de ejercer "control" sobre su vida y su comunidad para mejorar su bienestar y calidad de vida (Rappaport, 1987; Montero, 2004; Casique, 2010). En el caso de las mujeres, según Rowlands (1997), su empoderamiento tiene tres dimensiones: personal, de relaciones cercanas y colectivo. En lo personal, el empoderamiento implica la adquisición de habilidades como potencial transformador (Riaño, 2008). En las relaciones cercanas, implica el proceso por el cual las mujeres ganan control sobre los recursos intelectuales y materiales, y desafían la ideología del patriarcado y la discriminación por género (Erazo et al. 2014; Deere \& León, 2000; Pérez, 2001). Por último, en el nivel colectivo, el empoderamiento alude a la incidencia de las mujeres en las relaciones construidas al interior de un grupo y a su acción como colectivo para transformarse a sí mismas y a su entorno (Montero, 2004). Para las mujeres que participan de una organización, el empoderamiento colectivo implica transformar su percepción en la conciencia de sí misma, en las relaciones cotidianas y en la generación de ingresos (Alberti, 1998).

La relación entre el empoderamiento y los programas de emprendimiento parece paradójica. Así, aún cuando los programas con enfoque GED implicaron un avance en el reconocimiento de las desigualdades transversales de poder entre hombres y mujeres, según Campaña (1992), en lo general, los programas de emprendimientos han continuado viendo a las mujeres como las principales responsables del trabajo doméstico y "ayuda" económica en el hogar. De esta manera, los programas promueven una cierta autonomía de género de las mujeres, mientras que paradójicamente favorecen la reproducción de las inequidades, entre éstas, la doble jornada de trabajo (García \& Granados, 1997). Posiblemente por esto, frecuentemente, las participantes de estos programas no son capaces de cuestionar las relaciones de poder y subordinación ni de exigir una redistribución de roles más equitativa con sus parejas (Hidalgo, 2002; Riaño, 2008; Pérez, 2001; Cornejo et al. 1999).

La presente investigación enfocó la relación entre programas de emprendimiento y empoderamiento de las mujeres mediante la comparación de las experiencias de dos grupos de mujeres participantes de dos programas con enfoques diferentes: uno de emprendimiento económico, implementado bajo la perspectiva de género o enfoque GED, en Chile, y otro de emprendimiento económico, implementado sin perspectiva de género, es decir, con enfoque MED. La intención del estudio fue aportar de esta manera a la generación de conocimientos que clarifiquen si las características de uno u otro programa se condicen con consecuencias -a nivel personal, familiar y colectivo- de parte de las mujeres, las cuales podrían leerse o no en términos de empoderamiento. 


\section{METODOLOGÍA}

Se trató de un estudio de carácter cualitativo con enfoque intercultural y con estrategia metodológica comparativa. El método comparativo permitió, en el contexto de un estudio cualitativo, analizar los dos casos de estudio y buscar posteriormente ejes transversales que permitieran la comparación entre ambos (Tonon, 2011). De este modo, en el primer caso, se buscó describir las consecuencias personales, familiares y colectivas que tiene para las mujeres indígenas mapuche participar en un emprendimiento productivo que se implementa en el marco de un programa GED; y, en el segundo caso, se buscó la misma información en mujeres indígenas mayas participantes de un emprendimiento productivo que se realiza en el contexto de un programa MED. En seguida, a partir del análisis inicial de ambos casos, se exploró si en las descripciones que realizaban las mujeres, se observaban cuestionamientos a las inequidades de género, los que pudieran relacionarse con la implementación de su emprendimiento en el marco de un programa GED o MED.

Tabla 1. Variables del estudio de casos. Fuente: Elaboración propia.

\begin{tabular}{|l|l|l|}
\hline Variable constante & Variable no constante & Fenómeno de estudio \\
\hline $\begin{array}{l}\text { Programa orientado a1 } \\
\text { emprendimiento de mujeres } \\
\text { indigenas. }\end{array}$ & $\begin{array}{l}\text { Programa con perspectiva de género, GED: } \\
\text { Se capacita a las mujeres para que } \\
\text { desarrollen y gestionen por sí mismas un } \\
\text { emprendimiento productivo, a la par que se } \\
\text { promueve que cuestionen las inequidades de } \\
\text { género. }\end{array}$ & $\begin{array}{l}\text { Descripciones de las mujeres } \\
\text { respecto a las consecuencias de } \\
\text { participar en un emprendimiento } \\
\text { productivo y evidencias en estas } \\
\text { descripciones de cuestionamiento } \\
\text { a las inequidades de género. }\end{array}$ \\
\cline { 2 - 2 } & $\begin{array}{l}\text { Versus } \\
\text { Programa sin perspectiva de género, } \\
\text { MED: Se capacita a las mujeres para } \\
\text { que desarrollen y gestionen por sí } \\
\text { mismas un emprendimiento } \\
\text { productivo, pero no se promueve de } \\
\text { manera intencionada que cuestionen } \\
\text { las inequidades de género. }\end{array}$ & \\
\end{tabular}

Para relacionar los cuestionamientos a las inequidades de género y la implementación del emprendimiento bajo un programa GED o MED, de acuerdo a Lijphart (1971), se identificaron variables constantes y no constantes entre los casos, siendo las variables no constantes aquellas que se compararon entre sí, tal como se muestra en la Tabla 1.

Adicionalmente, la metodología se implementó con un enfoque intercultural, lo que permitió entender que los programas de emprendimiento son espacios interculturales que, al ser implementados por agencias externas a las comunidades de las mujeres participantes, corren el riesgo de modificar los significados propios de desarrollo económico de los pueblos indígenas (Rodríguez \& Iturmendi, 2013; Estermann, 2014). Se asumió este enfoque para 
entender los significados que las participantes le otorgan a su emprendimiento productivo, en estrecho diálogo con el mundo técnico representado por los programas estudiados. Como se verá en los resultados, este enfoque fue útil sobre todo para dar cuenta de las consecuencias a nivel colectivo que para las mujeres tiene participar en un programa de emprendimiento.

Técnicas de recolección y procedimiento de análisis

Para la recolección de datos, se utilizó la técnica de entrevista semi-estructurada. La entrevista semi-estructurada se consideró la más adecuada para acceder a las vivencias y percepciones de las participantes (Patton, 1990), indagándose en la percepción de las mujeres respecto a las consecuencias del emprendimiento sobre su vida personal, familiar y colectiva. Se entrevistó un total de 10 mujeres, 5 mapuche y 5 mayas. Todas las entrevistadas firmaron un consentimiento informado, donde se garantizaron sus derechos tales como, la voluntariedad de la participación, el derecho a la información y la seguridad del manejo confidencial y anónimo de la misma. El procedimiento de las entrevistas se implementó a través del contacto individual con las participantes de ambos programas. El acceso a las participantes fue facilitado por personal técnico y directivo tanto del programa GED como del programa MED cuyas consecuencias fueron estudiadas. En la siguiente tabla se presenta una descripción de las características etarias, familiares y socio-educativas de las entrevistadas.

Tabla 2. Características de las mujeres entrevistadas. Fuente: Elaboración propia.

\begin{tabular}{|c|c|c|c|c|c|c|}
\hline $\begin{array}{c}\text { No. de } \\
\text { entrevistada }\end{array}$ & Edad & Estado civil & $\begin{array}{c}\text { No. de hijo/ } \\
\text { s }\end{array}$ & $\begin{array}{c}\text { Pueblo } \\
\text { Indigena }\end{array}$ & $\begin{array}{c}\text { Escolaridad } \\
\text { programa en }\end{array}$ \\
\hline 1 & 48 & Casada & 6 & Mapuche & Básica incompleta & 2 años \\
\hline 2 & 53 & Casada & 6 & Mapuche & Básica incompleta & 2 años \\
\hline 3 & 29 & Conviviente & 1 & Mapuche & Media completa & 2 años \\
\hline 4 & 25 & Conviviente & 2 & Mapuche & Media incompleta & 2 años \\
\hline 5 & 24 & Casada & 0 & Mapuche & Técnico superior & 2 años \\
\hline 6 & 51 & Casada & 3 & Maya & Media incompleta & 4 años \\
\hline 7 & 30 & Casada & 2 & Maya & Media incompleta & 5 años \\
\hline 8 & 31 & Casada & 2 & Maya & Media incompleta & 6 años \\
\hline 9 & 37 & Casada & 4 & Maya & Media incompleta & 4 años \\
\hline 10 & 37 & Casada & 3 & Maya & Media incompleta & 4 años \\
\hline
\end{tabular}


En relación al análisis de la información, en un primer momento éste se realizó conforme al procedimiento de la Teoría Fundamentada (Strauss \& Corbin, 2002), cuyo objetivo fue desarrollar teoría basada en datos empíricos (Hernández et al. 2006). En este punto, se codificaron línea por línea las entrevistas transcritas con ayuda del programa Open Code 4.02 (2013). En este primer momento, conforme al método comparativo, los datos provenientes de cada uno de los casos se analizaron por separado (Tonon, 2011). La codificación teórica, resultado del proceso de análisis, permitió describir las consecuencias de participar en un emprendimiento productivo para las mujeres indígenas, mapuche y mayas, a partir del desarrollo de un proceso general, expuesto con sus subprocesos para ambos casos, en la siguiente tabla:

Tabla 3. Consecuencias de la participación en un emprendimiento productivo en mujeres indígenas*. Fuente: Elaboración propia.

\begin{tabular}{|c|c|}
\hline Grupo 1: Mujeres mapuche & Grupo 2: Mujeres mayas \\
\hline \multicolumn{2}{|c|}{ Proceso general: } \\
\hline \multicolumn{2}{|c|}{$\begin{array}{l}\text { Empoderarse como mujeres indígenas desde la demanda de mayor autogestión del } \\
\text { emprendimiento }\end{array}$} \\
\hline Subprocesos: & Subprocesos: \\
\hline a Re-experimentar problemas socioeconómicos & a Enfrentar problemas socioeconómicos \\
\hline b Recuperar la identidad cultural mapuche & b Reconocer su identidad cultural maya \\
\hline c Autovalorarse como mujeres mapuche & c Administrar las ganancias monetarias \\
\hline d Mejorar sus relaciones familiares & d Cambiar la cultura familiar machista \\
\hline e Desarrollarse integralmente & e Autovalorarse y desarrollarse como mujer \\
\hline
\end{tabular}

*Categorías resultado del análisis de información basado en la teoría fundamentada.

A partir de la codificación teórica expuesta en la Tabla 3, en un segundo momento del análisis y, de acuerdo al método comparativo (Tonon, 2011), se procedió a comparar los casos de estudio a partir de las variables identificadas en la tabla 1. El resultado de la comparación permitió concluir acerca de la relación entre las consecuencias de participar en un emprendimiento, el cuestionamiento a las inequidades de género por las mujeres y la situación del emprendimiento en cuanto a su implementación en el contexto de un programa GED o MED.

El proceso y subprocesos que explican las consecuencias de la participación en los emprendimientos productivos para las mujeres mapuche y mayas, presentados en la tabla 3 , se describen en el siguiente apartado. Posteriormente, se realiza la comparación de los casos y se presentan la discusión y las conclusiones de la investigación. 
G. MORA et al.

Empoderamiento como mujeres Mapuche desde la demanda de mayor autogestión del emprendimiento

Como se observa en la tabla 3, los resultados que se obtuvieron a partir del análisis de la información proporcionada por las mujeres mapuche, muestran las consecuencias de su participación en el emprendimiento, a partir de una experiencia de empoderamiento que, no obstante, demanda mayor autogestión, tal como se explica en seguida.

En primer lugar, las mujeres mapuche refieren que participar del emprendimiento productivo representó para ellas un nuevo intento para superar las carencias familiares socioeconómicas; es decir, las mujeres toman parte del emprendimiento productivo, pero tienen la duda de si esta iniciativa será un intento más de los que han realizado antes con poco éxito para superar la pobreza. A lo anterior se suma que tampoco cuentan con suficiente transporte y caminos para acceder a los centros de capacitación, de producción o de distribución de los productos que se generan en el emprendimiento. Por ello, emprender les exige importantes esfuerzos personales para asistir a las capacitaciones u otras instancias del emprendimiento productivo.

Además, las entrevistadas, comentan que el programa no se vincula con su vida en el territorio mapuche. Refieren que son mapuche lafquenche o gente de mar. Al respecto, señalan que con el emprendimiento les gustaría rescatar actividades propias de la economía mapuche, tales como la explotación de productos del mar, la venta de tejidos a telar o de productos basados en conocimientos medicinales de sus ancestros. Sin embargo, los técnicos del programa sólo les ofrecieron el rubro de crianza y comercio de ovinos, actividad que se vieron forzadas a aceptar para participar, pero que no forma parte de sus intereses como comunidades mapuche. Sobre esto, una entrevistada menciona: "Yo pienso que lo que falta acá en la comunidad... falta una empresa, una cooperativa para elaborar el ulte, el cochayuyo que sale del mar y todo eso".

De alguna manera, esta falta de concordancia entre la opción productiva del programa y los intereses de las participantes como mujeres mapuche limita sus motivaciones para participar del emprendimiento. Este último es experimentado por las participantes con melancolía, es decir, como una oportunidad que no favorece el fortalecimiento de sus tradiciones que, como comunidades indígenas, han ido perdiendo. Entre los relatos, una participante menciona: "Mi abuela hacía telar, ella tejía maravilloso (...) ¡cómo poder ir a buscar eso, volver a tenerlo!". No obstante que, en este sentido, el emprendimiento no satisface las expectativas de las mujeres, ellas intentan hacer del emprendimiento una oportunidad para recuperar la identidad cultural mapuche. Esto lo logran cuando ven en la crianza y venta de ovinos, un medio para retomar la herencia de sus abuelas como mujeres comerciantes que, en este caso, salen a vender sus ovejas. De esta manera, en el emprendimiento, las participantes se reconocen así mismas como mujeres mapuche, trabajadoras, luchadoras y comerciantes. 
En estrecha relación con lo anterior, a través del trabajo se potencia en las participantes un proceso de autovaloración como mujeres mapuche. Las mujeres enfatizan que el emprendimiento no sólo les permite mejorar su economía familiar sino, además, fortalecer su autoestima y desarrollo personal, a partir de lo cual se sienten más seguras de sí mismas y con mayor autovaloración. Al respecto, una participante señala: "Ahora tengo algo propio, algo mío, antes no tenía y ahora sí tengo mis ovejitas y así uno siente que puede lograr algo por uno". Es decir, el emprendimiento se convierte en un medio de reafirmación personal.

Para las mujeres mapuche, es importante valorarse a sí mismas porque, de acuerdo a las entrevistadas, al mejorar su autopercepción, se sienten más capaces de mejorar sus relaciones familiares. Las mujeres relacionan su participación en el emprendimiento con una mejora en sus relaciones familiares y se muestran orgullosas de recibir el apoyo y la admiración de sus respectivas parejas. Dicen sentirse más respetadas por éstas y, aunque reconocen que eso igual sucedía antes de empezar el proyecto productivo, no dudan en mencionar que ahora es mucho más notorio. Comentan con orgullo que el emprendimiento ha contribuido a cambiar la distribución de las labores domésticas, pues ahora ellas reciben "ayuda" en las labores de casa y, cuando tienen reuniones y capacitaciones del programa, son los esposos e hijos quienes asumen tareas domésticas que antes sólo hacían las mujeres. Por otra parte, la idea de percibir dinero y compartir gastos de la casa para apoyar a sus maridos genera gran satisfacción, tanto en ellas como en sus parejas. Al respecto, dice una entrevistada: "Yo creo que si él no me hubiera apoyado no me atrevería [a hacer el emprendimiento], y para mí es importante su apoyo, él me anima y también ayuda”.

Finalmente, desarrollarse integralmente constituye lo que las participantes señalan como una experiencia integral de desarrollo en tanto mujeres mapuche. Ellas sienten que con el emprendimiento potencian habilidades que creían no tener. Coinciden en que son capaces de resolver situaciones nuevas en relación con la actividad productiva que están emprendiendo. Pertenecer a un grupo de mujeres emprendedoras también es parte de ese proceso; en el grupo, comparten experiencias y refuerzan conocimientos en relación a su negocio. Ellas destacan que aspiran a una mejor calidad de vida para sus hijos y que capacitarse también las motiva, pues ponen a prueba sus capacidades y la necesidad de aprender más. Con el emprendimiento, las participantes desarrollan nuevas metas, que van más allá de la mera superación de carencias económicas. Sus motivaciones van cambiando y algunas, incluso, quieren regularizar sus estudios, superarse no sólo por los hijos, sino por ella mismas. "Sí, me veo diferente (...) veo mis corderitos y da gusto", "yo quiero terminar mis estudios básicos, ya estuve tiempo atrás en unos cursos para terminar la básica", son los testimonios de dos de las entrevistadas.

Tomados en su conjunto, estos cinco subprocesos dan cuenta de un empoderamiento de las participantes como mujeres mapuche, que tiene lugar mediante su participación en el emprendimiento. Para ellas, el empoderamiento significa mostrar frente a su grupo familiar que el propósito de mejorar su calidad de vida no implica sólo el plano económico, sino 
también tener un rol más protagónico en sus familias y en sus comunidades. Además, el emprendimiento es asociado por las mujeres con una oportunidad para reconocer sus propias capacidades. Sin embargo, la experiencia del empoderamiento deja entrever una demanda frente al programa: el de mayor capacidad de autogestión, lo que se observa sobre todo cuando las mujeres refieren que hubieran preferido un rubro productivo acorde a sus valores y tradiciones indígenas. Como dice una entrevistada: "Yo no me hallo en un lugar donde no hay mar... Siempre conversamos con la familia: 'tenemos que hacer algo pal turismo [en la playa, frente al mar] '. Pensamos vender café, y nos va a llegar una casa de subsidio y la instalamos para alojamiento, huevitos de campo, allá la cocinería [para los turistas en la playa]".

Empoderamiento como mujeres Mayas desde la demanda de mayor autogestión del emprendimiento

Como se observa en la tabla 3, los hallazgos encontrados en las mujeres mayas se expresan también a partir de cinco subprocesos principales, que fueron agrupados bajo el gran proceso de empoderamiento como mujeres mayas que, al igual que las mujeres mapuche, demandan mayor autogestión del emprendimiento, tal como se expone a continuación.

En primer término, las mujeres mayas señalan que con el emprendimiento enfrentan problemas socioeconómicos importantes. Con esto, ellas se refieren a su precaria condición socioeconómica y a las dificultades que experimentan para acceder a un trabajo remunerado que sea compatible con sus labores domésticas y el cuidado de los niños. Para hacer frente a la pobreza, consideran que el apoyo del gobierno y de otros organismos de la sociedad civil es fundamental. Al respecto, dice una entrevistada: "Cobro lo de PROSPERA [programa gubernamental para la superación de la pobreza], [el dinero] lo guardaba, cosas que necesiten mis hijas lo agarro de ahí [del dinero que guardé]".

De esta manera, las participantes ingresan al programa a partir de su necesidad de ayuda por parte del gobierno u otras fundaciones, sin distinción específica entre uno y otras. Consideran que la ayuda es una opción para cubrir necesidades primarias, tal como dice una entrevistada: "No hemos podido hacer una casita por falta de dinero". La carencia de recursos económicos se agrava, señalan, cuando la pareja tiene problemas con el consumo del alcohol o cuando el nivel escolar de las mujeres es mínimo. Ambas condiciones se presentan con frecuencia y, por consiguiente, los emprendimientos son una opción atractiva para las participantes.

A diferencia de las mujeres mapuche que establecen una relación con el emprendimiento en la discusión de si a través de éste recuperan o no su cultura, las mujeres mayas reconocen su identidad, principalmente, porque continúan usando la lengua, vistiendo en su mayoría el tradicional hipil y utilizando hierbas medicinales para diversos malestares. Asimismo, reconocen que actividades tales como coser, bordar, tortear, saber de crianza y el trabajo del campo son conocimientos ancestrales. Estos conocimientos, que forman parte de la cultura 
maya, se experimentan en lo cotidiano, por lo que ellas no relacionan el emprendimiento con una recuperación o valoración de su identidad cultural. "Sí me gusta también ser maya", señala una de las entrevistadas, sin que en su discurso se observen relaciones entre su experiencia como mujer maya y el emprendimiento. En este sentido, el rescate cultural de su pueblo no constituye un motivo fundamental para que las mujeres se incorporen y participen del emprendimiento.

Por el contrario, identifican como favorecedores de su participación en la actividad emprendedora otros factores como la religión. El hecho de que las parejas de muchas de estas mujeres se rehabiliten del alcoholismo al ingresar a una religión, por ejemplo, la evangélica, favorece que la mujer ingrese al trabajo productivo. En términos concretos, esto quiere decir que las parejas, cuando dejan de beber, "autorizan" a las mujeres a participar de las actividades del emprendimiento. "Entonces [mi marido] entró a un templo... A partir de eso cambió, dejó de tomar, pero cuando venía [yo] de trabajar [en el emprendimiento] estaba molesto", dice una de las participantes, evidenciando el control que su pareja ejerce sobre ella.

En este ambiente, marcado por el control que las parejas ejercen sobre las mujeres, ellas son, sin embargo, quienes ocupan el rol de administradoras de los recursos del hogar. Las mujeres administran las ganancias del esposo, ya que es costumbre que éstos les entreguen el sueldo. Sin embargo, con el ingreso del emprendimiento, las mujeres mayas dejaron de depender en su totalidad de la economía de la pareja; ahora, administran también su propio dinero. Esto último lo hacen con satisfacción, como se observa en el siguiente testimonio: "Lo necesitábamos, ahora estamos bien...tener mi propio dinero y no depender tanto de mi esposo". Para ellas, las ganancias superan sus expectativas económicas, además de que el emprendimiento representa la posibilidad de tener un trabajo remunerado, como dice una entrevistada: "Qué bueno que [el programa] llegó, porque aquí puedo tener un trabajo estable en mi casa".

La administración del dinero implica tomar decisiones para pagar la alimentación familiar, la educación de los hijos, las medicinas, los gastos asociados a la vivienda y, eventualmente, algún bien de tipo suntuario. Como los ingresos del emprendimiento son sustanciosos para el precario nivel de vida de las mujeres, lo ganado les alcanza hasta para "tener ahorritos" individualmente o con la pareja. Las ganancias en este sentido les permiten desarrollar cierta autonomía económica. No obstante, la precariedad económica de las mujeres se mantiene, ya que las ganancias están supeditadas a la cantidad de pedidos que reciben o, incluso, a su estado de salud o de sus hijos, porque si llegan a enfermarse no pueden producir y, por lo tanto, no generan ingresos.

Por otro lado, por participar en el emprendimiento, las mujeres mayas enfrentan críticas de sus parejas u otros familiares que ellas identifican como machistas. Una de las entrevistadas menciona: "Me reclamaban que descuidaba a mis hijos, que los dejaba mucho tiempo, pero yo les expliqué que no es por querer [trabajar], sino porque lo necesito". 
Muchas de las agresiones que viven las participantes de este estudio fueron provocadas por prejuicios respecto de su trabajo, tal como la desconfianza de sus parejas que piensan que, por ejemplo, las mujeres buscan amantes cuando trabajan. Las mujeres entrevistadas sobrellevan la subyugación hacia la pareja por medio de postergar sus necesidades y deseos personales para enfocarse en los hijos e hijas. No obstante, con el emprendimiento, ellas ejercen un trabajo remunerado, lo que significa "desobedecer al marido" y aceptar los insultos y amenazas que éstos ejercen sobre ellas para continuar trabajando. Algunas mujeres, logran cambios importantes en su dinámica familiar, tales como, que el esposo se adapte a la nueva situación y ayude en la crianza y algunas labores domésticas. Uno de los reconocimientos más gratificantes que se observa en ellas es que algunos maridos han aprendido a reconocer la importancia de su aporte económico.

Finalmente, las mujeres mayas indican reconocerse como sujetos de derechos en el emprendimiento. Consideran que, a pesar de vivir en una familia machista, tienen igualdad de derechos como los hombres y sienten la necesidad de decidir por sus vidas y de abandonar al marido en caso de ser violentadas. Aunque en condición de pobreza y subyugadas en una dinámica familiar donde la pareja masculina las controla, por otra parte, las mujeres señalan que en el emprendimiento tienen el reconocimiento de sus pares mujeres, los equipos técnicos y, eventualmente, del marido. De alguna manera, logran mayor autovaloración y desarrollo como mujeres. Desempeñar labores productivas les permite aprender técnicas nuevas, crear expectativas respecto de la posibilidad de ingresar a otros trabajos y adquirir habilidades que les permitan vender sus productos.

En suma, la participación en el emprendimiento trae satisfacción a las mujeres mayas y una experiencia de empoderamiento porque se sienten capaces en el trabajo y con libertad económica para tomar decisiones dentro del hogar. Como dice una entrevistada: "El trabajo, significa mucho para mí, es algo bueno. En ese aspecto yo he cambiado igual, ahora me siento más positiva con más animo de trabajar". Sin embargo, la experiencia de empoderamiento no obsta para que no demanden de alguna manera una mayor capacidad de autogestión de su emprendimiento. Al respecto dice una de las mujeres: "Es verdad que nuestros trabajos los llevan [a vender por medio de los equipos técnicos y las redes de distribución del programa] por todas partes, a New York, México y otros lugares (...), pero lo que yo le decía que nosotras (...) sería bueno que al menos una vez vaya una o dos de nosotras para que igual aprenda a hablar con el cliente, a conocer el cliente (...)". Con estas palabras, la entrevista alude a una demanda de mayor participación en la gestión de su negocio, que le permita salir adelante en el comercio de sus productos de manera autónoma.

\section{Comparación de los casos de estudio}

A continuación, se realiza el análisis comparativo de las descripciones que las mujeres mapuche y mayas hacen de las consecuencias de su participación en emprendimientos 
productivos y la evidencia en estas descripciones de cuestionamientos a la inequidad de género que pudieran relacionarse con la situación de implementación del emprendimiento bajo un programa GED o MED. En la siguiente tabla, se presenta de nueva cuenta, información contenida en la tabla 1, referida a aquellas variables constantes que, en este estudio, se consideraron en relación con el emprendimiento, implementado en el contexto de un programa con o sin perspectiva de género. En la misma tabla se agrega el proceso resultante del análisis de las entrevistas, el cual refiere a las consecuencias de participar en el emprendimiento, tanto para mujeres mapuche como mayas.

Tabla 4: Comparación de los casos de estudios. Fuente: Elaboración propia.

\begin{tabular}{|l|l|l|}
\hline Variable constante & Variable no constante & Variable dependiente: \\
\hline & $\begin{array}{l}\text { Programa con perspectiva de } \\
\text { género, enfoque GED } \\
\text { Prog r a m a or i e n t a d o a 1 } \\
\text { emprendimiento de mujeres } \\
\text { indígenas }\end{array}$ & $\begin{array}{l}\text { Consecuencias de la participación } \\
\text { de cuestionamiento a las } \\
\text { inequidades de género }\end{array}$ \\
\cline { 2 - 2 } & Versus & $\begin{array}{l}\text { Empoderarse como mujeres } \\
\text { indígenas y desde la demanda de } \\
\text { m a y or a u t o g e s t i ó n d e } 1 \\
\text { emprendimiento }\end{array}$ \\
\cline { 2 - 3 } & $\begin{array}{l}\text { Programa sin perspectiva de género, } \\
\text { enfoque MED }\end{array}$ \\
\hline
\end{tabular}

Como se observa en la tabla 4, la información recabada no señaló diferencias sustantivas en los procesos de empoderamiento de las mujeres de ambos pueblos indígenas. Por el contrario, en ambos casos las mujeres refieren experiencias de empoderamiento, acompañadas de demandas de mayor autogestión del emprendimiento. Sin embargo, las diferencias entre los casos podrían aludir a situaciones particulares del ámbito privado respecto a los modos en que se cuestiona la inequidad de género: así, mientras las mujeres mapuche refieren un cambio positivo en su posición familiar, expresado básicamente en términos del reconocimiento al interior de sus familias a partir de su participación económico-productiva, las mujeres mayas refieren un cambio también positivo a nivel familiar, pero acompañado de conflictos y control por parte de sus parejas, situaciones que identifican como expresiones del "machismo". Lo que llama la atención, asimismo, es que las mujeres mayas cuando reconocen de manera explícita el machismo en sus familias, expresan un cuestionamiento a la inequidad de género, situación que no aparece de manera evidente en los relatos de las mujeres mapuche. Esta situación resulta paradójica pues, justamente, el programa que impulsaría el reconocimiento de las inequidades de género, es el programa GED, del que participaron las mujeres mapuche. 


\section{DISCUSIÓN}

Los hallazgos dan cuenta de la relación entre las consecuencias de la participación de mujeres indígenas, mapuche y mayas, en emprendimientos productivos, y las evidencias de cuestionamientos a las inequidades de género, que potencialmente podrían relacionarse con la implementación del emprendimiento en el marco de un programa GED o MED. Para el caso de ambos grupos de mujeres, mapuche y mayas, las participantes mostraron un significativo empoderamiento a niveles personal y familiar; no así a nivel colectivo, donde son más bien las mujeres mapuche quienes se reivindican con fuerza como miembros de una comunidad lafkenche, mientras las mujeres mayas no relacionan de manera explícita su emprendimiento con el rescate de su identidad indígena. Además, el papel que juega el dinero en ambos casos parece ser diferente; así, mientras para las mujeres mayas el emprendimiento posibilitó que ellas obtuvieran ganancias económicas suficientes para sentir que enfrentan de manera satisfactoria sus necesidades socioeconómicas, en el caso de las mujeres mapuche más bien prevalece la duda respecto a las posibilidades del emprendimiento para salir de la pobreza.

Con base en lo anterior, es que los datos sugieren que el enfoque MED podría jugar un papel relevante como catalizador de cuestionamientos a la inequidad de género, por parte de las mujeres, sobre todo si éstas consideran que reciben ingresos que les permiten hacer frente a las necesidades personales y familiares básicas y tomar decisiones al respecto. Por el contrario, en el caso del emprendimiento de las mujeres mapuche, el enfoque GED se tradujo en cuestionamientos sutiles respecto a la inequidad de género, expresados por ejemplo en el orgullo de las mujeres al recibir la "ayuda” de los maridos en las labores domésticas, sobre todo cuando ellas están realizando alguna actividad propia del emprendimiento. Sin embargo, hay que mencionar que la ayuda que reciben de parte de las parejas o maridos parece cobrar sentido para las mujeres mapuche en un marco mayor de reivindicación cultural indígena. Con base en lo anterior, se piensa que el caso de las mujeres mapuche podría ilustrar con mayor fuerza que el caso maya la intersección y, quizá superposición, de la identidad indígena sobre la identidad de género.

Comparativamente, se resalta que ambos grupos de mujeres demandan mayor autogestión de sus respectivos emprendimientos. Este último punto es concordante con lo encontrado por Mendoza y Chapulín (2015) respecto a la inserción laboral de las mujeres y, a partir de ella su empoderamiento, el cual se relaciona con el nivel de autogestión de las mujeres respecto a su actividad productiva.

Tal como menciona Rowlands (1997), el empoderamiento se da a nivel personal, de relaciones cercanas, incluidas las familiares y de relaciones colectivas, incluidas las relaciones comunitarias. Si bien las mujeres mapuche se empoderan en las tres dimensiones, a diferencia de las mujeres mayas que, por motivos no discriminados en este estudio, parecen tener mayores consecuencias a nivel personal y familiar, por otra parte, las mujeres mapuche tienen una reivindicación en términos de género menos evidente. Esta última situación indica 
la necesidad de trabajar con las mujeres indígenas desde una perspectiva interseccional, es decir, una perspectiva que permita poner en juego tanto la identidad y desigualdad de las mujeres como indígenas como la identidad y desigualdad de ellas como propiamente mujeres (Lombardo \& Verloo, 2010).

\section{CONCLUSIONES}

Se concluye que, potencialmente, cuando se trabaja con mujeres indígenas, tanto un programa GED como uno MED pueden tener efectos de empoderamiento. No obstante, es posible que los sentidos que las mujeres le otorguen al empoderamiento dependa del cruce de identidades y experiencias entre sus identidades culturales indígenas y sus identidades de género. Por otro lado, el estudio señala con mayor claridad que la autogestión es un elemento considerado relevante por las mujeres indígenas involucradas en un emprendimiento productivo.

Se sugiere que futuras investigaciones sigan comparando ambos tipos de programa y sus consecuencias sobre los niveles de empoderamiento personal, familiar y colectivo de las mujeres. Sería conveniente profundizar en la relación entre las características del programa en el que tiene lugar el emprendimiento y aquellos elementos culturales que las mujeres indígenas parecen retomar en el marco de sus actividades productivas y que están en estrecha relación con sus formas de vida y reivindicaciones como miembros de comunidades indígenas.

Casi para terminar, se menciona que la validez del estudio se fortaleció a partir del procedimiento efectuado de triangulación de espacio que, según Pérez (1994), implicó reunir datos referidos al mismo tema a partir de varias culturas, en esta investigación, a partir de las mujeres mapuche y maya, con la intención de superar el riesgo de que los hallazgos se encontraran sesgados por provenir de un único tipo de sujeto de información.

\section{REFERENCIAS}

Alberti, P. (1998). La organización de mujeres indígenas como instrumento de cambio en el desarrollo rural con perspectiva de género. Revista Española de Antropología Americana, 28, 189-213.

Campaña, P. (1992). El contenido de género en la investigación en sistemas de producción. Santiago: Red internacional de metodología de Investigación de Sistemas de producción.

Canorea, N. (2015). UNIFEM: Defensa y promoción de la mujer y su derecho a la educación. Journal of Supranational Policies of Education, 3, 83-100.

Casique, I. (2010). Factores de empoderamiento y protección de las mujeres contra la violencia. Revista Mexicana de Sociología, 72(1), 37-71.

Castelnuovo, N. (2013). Mujeres indígenas y desarrollo: las experiencias de tres mujeres guaraníes del noroeste argentino. Cuicuilco, 20(57), 11-46.. 
Cornejo, E., Aranda, P., \& Denman, C. (1999). Reseña de "Poder y empoderamiento de las mujeres” de Magdalena de León (compiladora). Región y Sociedad, XI. Recuperado de http://www.redalyc.org/articulo.oa?id=10201808

Deere, C., \& León, M. (2000). Género, propiedad y empoderamiento: Tierra, Estado y mercado en América Latina. Bogotá: Tercer mundo S.A.

Erazo, M., Jiménez, M., \& López, C. (2014). Empoderamiento y Liderazgo Femenino; su papel en la autogestión comunitaria en el corregimiento El Hormiguero-Valle Del Cauca. Avances en Psicología Latinoamericana, 32(1), 149-157.

Estermann, J. (2014). Colonialidad, descolonización e interculturalidad: Apuntes desde la Filosofía Intercultural. Polis, 13(38), 347-368.

Fostner, K. (2013). La artesanía como estrategia de desarrollo rural: el caso de los grupos de artesanas en la región de Puno (Perú). Cuadernos de Desarrollo Rural, 10(72), 141-158.

García, E. (2008). Políticas de Igualdad, Equidad y Gender Mainstreaming. ¿De qué estamos hablando? Marco Conceptual. San Salvador: PNUD y Genera.

García, F., \& Granados, A. (1997). Educación: ¿integración o exclusión de la diversidad cultural? Granada: Laboratorio de Estudios Interculturales, Universidad de Granada.

Hernández, R., Fernández, C., \& Baptista, P. (2006). Metodología de la Investigación. México: McGrawHill.

Hidalgo, N. (2002). Género, empoderamiento y microfinanzas. Un estudio de caso en el norte de México (Tesis de maestría). Recuperado de http://cedoc.inmujeres.gob.mx/ documentos_download/100852.pdf

Hoinle, B., Rothfuss, R., \& Gotto, D. (2013). Empoderamiento espacial de las mujeres mediante la Economía Solid. Cuadernos de Desarrollo Rural, 10(72), 117-139.

IICA (1993). Guías para la formulación de proyectos de inversión agrícola con enfoque de género. Proyecto Género, mujer y desarrollo, Programa III. San José: Mimeo.

Kabeer, N. (1999). Resources, agency, achievements: reflections on the measurement of women's empowerment. Development and Change, 30, 435-464.

Liphart, A. (1971). Comparative Politics and the Comparative Method. The American Political Science Review, 65(3), 682-693.

Lombardo, E., \& Verloo, M. (2010). La “interseccionalidad” del género con otras desigualdades en la política de la Unión Europea. Revista Española de Ciencia Política, (23), 11-30.

Mendoza, M., \& Chapulín, J. (2015). Turismo, trabajo femenino y empoderamiento de las mujeres en bahías de Huatulco, Oaxaca - México. Estudios y perspectivas en turismo, 24(2), 316-335.

Moser, C. (1991). La planificación de género en el tercer mundo: enfrentando las necesidades prácticas y estratégicas de género. En V. Guzmán, P. Portocarrero \& V. Vargas (Comp.), Una nueva lectura: Género en el desarrollo (pp. 55-124). Lima: Flora tristán ediciones.

Montero, M. (2004). Introducción a la Psicología Comunitaria. Desarrollo, conceptos y procesos. Buenos Aires: Editorial Paidós. 
Naciones Unidas. (2007). Declaración de las Naciones Unidas sobre los derechos de los pueblos indígenas Recuperado de http://www.un.org/esa/socdev/unpfii/documents/ DRIPS_es.pdf

Open Code (2013). Umeå University, Suecia: ICT Services and System Development and Division of Epidemiology and Global Health.

Patton, M. (1990). Qualitative Evaluation and Research Methods. California: Sarge Publications.

Pérez, G. (1994). II Técnica de Recolección de datos. Investigación Cualitativa. Retos e Interrogante. Madrid: La Muralla S.A.

Pérez, E. (2001). El proceso de empoderamiento de mujeres indígenas organizadas, desde una perspectiva de género. Estudios Agrarios (17), 125-169.

Rappaport, J. (1987). Terms of Empowerment/Exemplars of prevention: toward a theory for community psychology. American Journal of Community Psychology (15), 121-148.

Riaño, R., \& Okali, C. (2008). Empoderamiento de las mujeres a través de su participación en proyectos productivos: experiencias no exitosas. Convergencia, 15(46), 119-141.

Rodríguez, E., \& Iturmendi, A. (2013). Igualdad de género e interculturalidad: enfoques y estrategias para avanzar en el debate. Panamá: PNUD.

Rowlands, J. (1997). Empoderamiento y mujeres rurales en Honduras: un modelo para el desarrollo. En M. León (Comp.), Poder y empoderamiento de las mujeres (pp. 213-245). Bogotá: Facultad de Ciencias Humanas, TM Editores, Tercer Mundo.

Subramaniam, M. (2012). Grassroots groups and poor women's empowerment in rural India. International Sociology, 27(1), 72-95.

Strauss, A., \& Corbin, J. (2002). Bases de la investigación cualitativa. Técnicas y procedimientos para desarrollar la teoría fundamentada. Medellín: Contus.

Tonon, G. (2011). La utilización del método comparativo en estudios cualitativos en ciencia política y ciencias sociales. Kairos, Revista de Temas Sociales, 27, 1-12.

Young, K. (1997). El potencial transformador en las necesidades prácticas: empoderamiento colectivo y el proceso de planificación. En M. León (Comp.), Poder y empoderamiento de las mujeres (pp. 99-118). Bogotá: Facultad de Ciencias Humanas, TM Editores, Tercer Mundo. 
